Inspection Time and General Speed of Processing

Tess A. O’Connor and Nicholas R. Burns

Department of Psychology, University of Adelaide, South Australia

Running head: IT and Speed of Processing

Correspondence concerning this article should be sent to:

Nick Burns

Department of Psychology

University of Adelaide

South Australia 5005

Electronic mail: nick.burns@psychology.adelaide.edu.au

This is a post-print of an article published under the same title by Elsevier Ltd in the journal Personality and Individual Difference (vol. 35, no. 3, 2003, pp. 713-724). The published version can be found at:

http://www.elsevier.com/wps/find/journaldescription.cws_home/603/description\#description 


\begin{abstract}
Recent research suggests that the relationship between inspection time (IT) and psychometric intelligence arises because IT is a measure of general speed of processing (Gs). However, hierarchical models of intelligence propose several distinct speed of processing factors; this study examines IT in relation to these multiple speed factors. Participants $(N=102)$ completed tests of speed of processing yielding 18 measures. Factor analysis revealed a second order general speed factor (Gs) and four group factors: perceptual speed, visualisation speed, decision time and movement time. IT correlated with a visualisation speed factor $(r=.36)$ and with a perceptual speed factor $(r=.28)$. However, the correlation between IT and perceptual speed was near-zero when the correlation with visualisation speed was partialled out. These findings are consistent with the notion that IT is a measure of Gs but suggest that IT most directly measures speed of visualisation processes. These results are also congruent with research on the psychophysics of IT.
\end{abstract}




\section{Inspection Time and General Speed of Processing}

Inspection time (IT) is one of many so-called elementary cognitive tasks (ECTs) that have been studied in an attempt to explain some of the variance in psychometric intelligence (i.e., IQ). Many researchers have replicated the finding of a relationship between IT and IQ with three reviews (Grudnik \& Kranzler, 2001; Kranzler \& Jensen, 1989; Nettelbeck, 1987) putting the corrected correlation at about -.5 . Despite wide interest, however, researchers have not been able to resolve fully the issue of what IT is measuring (Deary, 2000).

A consistent finding of IT research has been that IT is more strongly related to performance IQ (PIQ) rather than with verbal IQ (VIQ) from the Wechsler scales (Kranzler \& Jensen, 1989; Nettelbeck, 1987). A common assumption was that PIQ measures fluid ability (Gf; Deary, 1993; Kline, 1991; Sattler, 1982) and, upon this basis, it was thought that IT measured Gf. Indeed, it was even proposed that IT may provide a culture-free test of intelligence (Brand \& Deary, 1982). However, recent research has rendered this latter interpretation untenable.

For example, a study by Crawford, Deary, Allan and Gustafsson (1998) located IT within the factor structure of the WAIS - R. Confirmatory factor analyses suggested three group factors and one general factor; these factors were all orthogonal. IT loaded on a perceptual organisation factor (-.39) but only weakly on the general factor (i.e., g). These findings led to the suggestion that IT was not related to ' $g$ ' directly but that it was related to the group factor perceptual organisation.

Orienting their research by the Horn-Cattell model of intelligence (Horn \& Cattell, 1966, Horn \& Noll, 1994), Burns, Nettelbeck and Cooper (1999) reported that IT correlated with a test of general speed of processing (Gs) but not with tests of Gf or general visualisation ability (Gv). This result has been replicated by Mackintosh and Bennett (2002) using different tests and a different participant population. Burns and Nettelbeck (in press), using a broad sampling of abilities tests, confirmed that IT provides a measure of Gs but does not measure Gf or Gv. Based on these studies, 
IT is most likely a marker of Gs, which suggests that the IT - IQ relationship may be mediated through a relationship between IT and Gs.

Recent models of intelligence paint a complex picture of speed of processing abilities (Carroll, 1993; Horn, 1988; Robert \& Stankov, 1999). Horn (1988; 1989; Horn \& Noll, 1994) extended Gf - Gc theory to include a speed factor called correct decision speed (CDS) derived from the speed with which people provide correct answers to a variety of tests. Stankov and Roberts (1997; Roberts \& Stankov, 1999) have proposed a hierarchical model of speed of processing with the most complex mental speed factor being test-taking speed (which is very similar to Horn's correct decision speed). Perceptual speed (PSp) was theorised to be the next most complex factor and is marked by tests such that, given enough time, almost anyone could complete all items correctly. At the next level of complexity was decision time (DT), which represents the time required to make a simple decision based on sensory information. At the lowest level in this hierarchy was movement time (MT) which is a psychomotor ability with minimal cognitive requirements. Stankov and Roberts $(1997$, p. 75$)$ remarked "these various speed factors tend to define a single factor at the highest order of analysis" but they also noted that the strength of this factor is often weak. Roberts \& Stankov (1999) included a general speed factor in their model. However, Horn (1988) and Carroll (1993) did not. Therefore, the status of a higher order general speed factor is debatable, being also clouded by uncertainty over the order of analysis at which such a factor might appear.

Studies attempting to locate IT within hierarchical models of cognitive abilities have so far shown IT as related to Gs. But given that Gs is factorially complex, it is worth re-examining the question of what IT is measuring. Beginning at the lowest level of complexity of the proposed speed hierarchy, it has been consistently shown that IT is independent of MT (Burns \& Nettelbeck, in press; Carroll, 1991; Kranzler \& Jensen, 1991). This follows because IT does not incorporate any psychomotor component. Concerning DT, it has been shown that both IT and DT share variance with IQ (Jensen, 1982; Nettelbeck, 1987) but the literature is unclear on whether they 
share common variance with IQ. Kranzler and Jensen (1991) administered a battery of ECTs to 101 university students and exploratory factor analysis revealed both DT and MT factors but IT did not load on either factor. Carroll (1991) reanalysed these data and found that IT loaded on a factor defined by choice DT but was independent of a factor defined by 'odd-man-out' reaction time (OMO DT; see Frearson \& Eysenck, 1986). Bates and Eysenck's (1993) study found the exact opposite; that is, IT was related to OMO DT but was independent of choice DT. Finally, Burns and Nettelbeck (in press) found that OMO DT loaded on Gf while IT loaded on Gs, suggesting IT does not share variance with DT. These four studies do not clarify the relationship between IT and DT but do suggest that IT may have differential relationships to choice DT and OMO DT.

Concerning the perceptual speed (PSp) factor, the studies reviewed above (Burns \& Nettelbeck, in press, Burns et al., 1999; Mackintosh \& Bennett, 2002) have essentially taken PSp tests as markers for Gs and have concluded that because IT is related to these tests, that IT provides a measure of Gs. Finally, there have been no studies which have examined the relationship of IT to a CDS factor.

This study therefore investigated the hierarchical structure of mental speed. The presence of a higher order factor (Gs) was tested and the location of IT within this hierarchy was investigated. IT was expected to provide a measure of perceptual speed (PSp) and to load on the higher order factor (Gs) via this path.

\section{Method}

\section{Participants}

Participants $(N=102)$ were 71 first year psychology students from the University of Adelaide and 31 people recruited from the wider community. Thirty-four participants were male (mean age $=22.0, \mathrm{SD}=5.9$ years) and 68 female (mean age $=19.7, \mathrm{SD}=4.6$ years)

\section{Test Materials}

Three tests of perceptual speed (PSp) were administered. (i) Digit Symbol, from the WAIS - R was administered following the instructions in the manual (Wechsler, 1981). (ii) A mental 
rotation task required that participants decide if two letters, or numbers, were identical or mirror images of one another; the second symbol had been rotated to a varying degree. (iii) A cross out task required that participants cross out the exact copy of a group of letters, or numbers, among four choices. For each of these tests, participants were given $90 \mathrm{sec}$ to complete as many items as possible and the measure taken was the number correct.

The IT stimulus was presented on a computer monitor approximately $0.5 \mathrm{~m}$ from the participant. The target figure consisted of two vertical lines $22 \mathrm{~mm}$ and $27 \mathrm{~mm}$ long joined at the top by a horizontal line, $12 \mathrm{~mm}$ long. The shorter line appeared on the left or right equiprobably. The target figure, preceded by a small white circle which acted as a warning cue, was presented for a short period and then followed by a "flash mask" (Evans \& Nettelbeck, 1993) that lasted $290 \mathrm{msec}$. The flash mask consisted of two vertical lines each $37 \mathrm{~mm}$ long and shaped like lightning bolts. Participants were required to indicate, via the keyboard, which line was shorter, the left or the right.

The apparatus for presentation and responding for choice reaction time (CRT) was as described by Jensen \& Munro (1979). The apparatus was scaled down to consist of a panel, $15 \mathrm{x}$ $24 \mathrm{~cm}$, painted white and tilted at a $30 \mathrm{deg}$ angle. Eight response keys (each with a corresponding red light) are arranged in a semi-circle around a home key. The participants were instructed to hold down the home key and, after a pseudo-random period of 1 to $4 \mathrm{sec}$, one of the other eight lights came on. The participant had to take their finger off the home key and push the appropriate response key as quickly as possible. Responses were divided into a decision time component (DT, the time the participant took to remove their finger from the home key after a light has come on) and a movement time component (MT, the time between removing finger from the home key and pressing a response key).

There were three conditions, which differed in the number of lights, either two, four, or eight, that needed to be monitored. Each task was repeated 60 times and the median DT and MT for correct responses were recorded. 
The display and response panel for odd-man-out reaction time (OMO) was the same as that used for CRT. For each presentation of the OMO task, three of the eight lights illuminated. The three lights were arranged so that the distance between the left light and the centre light was different to the distance between the right light and the centre light. This was explained to the participants as a pair of lights (the two closest together) and an 'odd-man-out' (that light that is further away). Following Frearson, Barrett and Eysenck (1988), 12 different displays (six being left-right mirror images of the other six) were chosen from the 44 alternative arrangements because they had been found to correlate most strongly with Raven's matrices scores. Each of the 12 displays was presented five times, to produce 60 trials. Both DT and MT for correct responses were recorded.

In the Triplets Numbers test (Wittenborn, 1943) participants were required to evaluate three digits according to one or more rules. Triplet 1: Press "YES" if the second number is the largest. Otherwise, press "NO". Triplet 2: Press "YES" if the second number is the largest and the third number is the smallest. Otherwise, press "NO". Triplet 3: Press "YES" if the first number is the largest and the second number is the smallest or if the first number is the smallest and the third number is the largest. Otherwise, press "NO". For each item, three digits were presented on the computer screen and participants had to press YES if they thought the digits matched the rule or NO if they did not. Each triplet presented had an equal probability of requiring a YES or NO response. Each section consisted of 24 items and participants had to complete the whole set. Correct decision speed was measured for each response, which led to three median correct decision speed measures (one for each section).

For the Swaps test (Crawford, 1988), participants were instructed mentally to swap the order of three letters presented on the computer monitor and then to indicate the final order of the letters. A choice of solutions were presented at the bottom of the screen, numbered 1 to 6 . There were three sections of the test, which differed in the number of swaps required (2, 3, or 4 swaps). Each section had 24 items and participants had to complete all items. This produced three median correct 
decision speed scores (one for each section). The stimulus for each item was a different set of three letters, such that the participant never repeated the same task.

\section{Procedure}

The test sessions were done in groups of up to four participants and lasted about $1.5 \mathrm{hrs}$. Firstly, participants completed the perceptual speed tasks in the following order: Digit Symbol (DS), mental rotation (MR) and cross out (CO). Each test was explained and participants completed practice items to ensure they understood the task requirements. The three tests took about 7 minutes to complete.

Next, the IT task was explained verbally followed by a series of practice trials to familiarise participants with the task. In order to move on to the estimation stage, participants had to obtain 10 correct trials out of 10 with a stimulus onset asynchrony $(\mathrm{SOA})=835 \mathrm{~ms}, 10$ correct trials out of 10 with $\mathrm{SOA}=418 \mathrm{~ms}$ and 9 correct trials out of 10 with $\mathrm{SOA}=250 \mathrm{~ms}$. All of the participants met these criteria. The estimation process began with $\mathrm{SOA}=250 \mathrm{~ms}$ and followed an adaptive staircase algorithm (Wetherill \& Levitt, 1965). The algorithm required three correct responses at any SOA before reducing the SOA by $18 \mathrm{~ms}$. When an incorrect response was made the SOA increased by $18 \mathrm{~ms}$. The average SOA was calculated over eight reversals of direction on the staircase giving an estimate of the SOA with an associated probability of $79 \%$ of making a correct response. The IT task took approximately 8 min to administer.

CRT was administered next following the procedure of Jensen and Munro (1979). Preceding each condition, subjects completed 10 practice trials, all of which had to be completed correctly, to familiarise themselves with the task. For the OMO task, completed after CRT, participants completed 20 practice trials, of which 18 had to be correct in order to move on to the task proper. Participants were instructed not to lift their finger from the home key until they had made a decision about which light was the target. Trials began when the participant pushed down on the home key. The RT tasks took about 20 min to administer. 
The Triplet Numbers task was presented next. The task was verbally explained to the participants along with instructions presented on the computer screen in front of them. One example was displayed and explained to the participant along with an explanation of the response keys. Participants were instructed to remember the rule because it was not shown at the start of each trial. Once the rule was learned, participants completed 12 practice trials of which 10 had to be completed correctly. This was followed by 24 test items. Participants went through the same process of practice and trials for the second and then the third set of triplets. The Triplets test took about 20 min to complete.

The Swaps test was presented last and it was explained both verbally and on the computer screen. The response keys were explained and both speed and accuracy were emphasised as being important. All of the practice for this test, four items of each type of which three had to be done correctly, was done at the start followed by the 72 test items. The items were presented randomly so that the participant did not know whether to expect a 2-swap, 3-swap, or 4-swap item on any trial. The Swaps test took between $15-35$ min.

Results

Table 1 shows the correlation matrix and descriptive statistics for the 18 variables in the battery. Of note are the significant correlations of IT with OMO DT but not with any of the CRT measures and the significant correlation of IT with both Digit Symbol and Mental Rotation and with Triplet 1, Triplet 2, and all the Swaps measures. Other correlations are consistent with expectations.

Insert Table 1 about here

Exploratory factor analyses were carried out following the procedures outlined by Carroll (1993, 1995; see also Burns \& Nettelbeck, in press). Eighteen variables were included in the factor analysis; the three perceptual speed variables were reflected prior to analysis. A five-factor orthogonal solution was well defined, there being at least two variables with their highest loadings 
on each factor. An oblique rotation was then performed on the five factor solution. Table 2 shows the structure loading matrix for this solution with the factors being interpreted as CDS, PSp, DT, MT and visualisation speed (VSp). The correlation matrix for these five factors is at the bottom of Table 2. It is worth noting the sizeable correlation between the CDS factor and the PSp factor.

Insert Table 2 about here

All five factors were considered acceptable for higher order analysis according to Kaiser's (1981) criterion and the matrix yielded one second order factor, interpreted as Gs. The loadings of the five factors on the second order factor are shown in Table 2; of particular note is that the Gs factor was near identical with the CDS factor.

The exploratory model shown in Table 2 was estimated using AMOS (Arbuckle, 1999). The loadings shown in bold defined the five first-order factors except that OMO DT was also allowed to load on the DT factor and Triplet 1 and Triplet 2 were also allowed to load on VSp and PSp, respectively; all five factors loaded on a second-order factor (Gs). This model provided an acceptable fit to the data $\left(\chi^{2}=153.8, \mathrm{df}=127, \mathrm{p}=0.053 ;\right.$ AGFI $=0.813 ; \mathrm{TLI}=0.974 ; \mathrm{RMSEA}=$ 0.046); however, both Triplet 1 and Triplet 2 had non-significant loadings on PSp and so these two paths were dropped from the model.

It had not escaped our attention, however, that the CDS factor may simply reflect common method variance associated with the Swaps measures; to address this possibility we examined the validity of a four factor model in which the CDS factor was dropped and an aggregated Swaps variable was used as an additional marker of PSp. This model provided a better fit to the data than the original model $\left(\chi^{2}=115.5, \mathrm{df}=99, \mathrm{p}=0.123 ; \mathrm{AGFI}=0.837 ; \mathrm{TLI}=0.978 ; \mathrm{RMSEA}=0.041\right)$. In order to confirm the location of IT within this hierarchy, the model was run again omitting IT and calculating scores on each of the factors. IT correlated significantly with factor scores for both $\operatorname{VSp}(r=.36, p<0.001)$ and with PSp $(r=.28, p<0.01)$. However, the partial correlation between 
IT and PSp, controlling for VSp, was near-zero $(r=.02, p=.83)$. Conversely, the partial correlation between IT and VSp, controlling for PSp, was statistically significant $(r=.23, p<.05)$.

The final model, then, is a four factor model consisting of PSp, VSp, DT and MT at the first level and a higher order Gs factor at the second level of analysis. This factorial structure of mental speed is very similar to that presented by Stankov and Roberts (1997; Roberts \& Stankov, 1999). Their model stated that MT, DT, PSp and CDS defined mental speed. It is evident that their first three factors are confirmed but that a true CDS factor did not emerge from this dataset. However, an additional first order speed factor, here interpreted as visualisation speed (VSp), also emerged. This factor subsumes the Triplets variables, OMO DT and IT. It is worth noting that although all three triplets variables loaded on VSp in the confirmatory analysis, they displayed loadings on both VSp and PSp in the exploratory analysis.

To summarise: IT correlated with a VSp factor and to a lesser degree with the PSp factor. However, the correlation between IT and PSp was near-zero when VSp was partialled out. This finding provides evidence that the relationship between IT and IQ is likely mediated by the relationship between IT and Gs, via the construct visualisation speed which can be distinguished from perceptual speed as measured by tests like Digit Symbol. However, the VSp and PSp factor appear to be closely related. This latter point explicates the results reported by Burns and Nettelbeck (Burns \& Nettelbeck, in press, Burns et al., 1999) and by Mackintosh and Bennett (2002).

\section{Discussion}

This investigation aimed to clarify the relationship between IT and IQ. Factor analysis of 18 speed of processing variables defined a model consisting of four narrow speed factors labelled: perceptual speed, visualisation speed, decision time and movement time. The model includes a second-order general speed factor. These factors are now briefly discussed.

Perceptual speed (PSp). This factor was defined mainly by Cross Out and Digit Symbol which had substantial loadings on this factor and near zero loadings on the other first-order factors. 
Mental Rotation had its highest loading on this factor but also had a non-trivial loading on the visualisation speed factor (see below). Triplet 1 had similar loadings on PSp and VSp in the exploratory analysis but the confirmatory analysis suggested Triplet 1 was more related to the VSp factor. The Swaps aggregate score had a moderate loading on the PSp factor. To the extent that this factor was defined primarily by classic clerical speed tests, it is reasonable to label this as a perceptual speed factor.

Visualisation speed (VSp). This factor is interpreted as representing the time needed to perform tests requiring somewhat complex visualisation of stimuli. Confirmatory factor analysis suggested all three Triplets variables defined this factor. OMO DT has its highest loading on this factor and a substantial loading on the DT factor (see below). IT also had its highest loading on this factor but near-zero loadings on the other first order factors. Partial correlation analysis confirmed that IT is more related to VSp rather than PSp. As already noted, Mental Rotation also had a substantial loading on this factor.

Decision time (DT). The choice DT variables defined this factor and OMO DT had a secondary loading on this factor. This factor is interpreted as the time required to make a simple decision based on sensory information. Interpretation of this factor is not controversial but it is acknowledged that the factor is very narrowly defined by this battery.

Movement time (MT). This factor is derived from reaction time tests that attempt to isolate movement time from decision time. All measures of MT defined this factor. It is worth noting that the DT and MT variables uncovered in this investigation were not independent from one another.

General speed of processing (Gs). This is the general factor of this battery. It represents the speed at which people perform tasks ranging from somewhat complex cognitive tasks to simple sensory discriminations. From Table 2 it can be seen that the loadings of the first order factors on Gs reflect the hypothesised complexity of processing required by the tasks that define those factors.

Having discussed the nature of the outcomes of the factor analysis reported here, the question of the relation of IT to these factors can be addressed. The results are then discussed with 
respect to earlier studies. Previously, IT has been shown to be a measure of general speed of processing (Burns \& Nettelbeck, in press, Burns et al., 1999, Mackintosh \& Bennett, 2002). In this study, IT was related to visualisation speed, which is thought to measure the speed at which one visualises an image on which a decision must be made. Vickers and Smith (1986, p.609) defined IT as "the time required by a subject to make a single observation or inspection of the sensory input on which a discrimination of relative magnitude is based". Even more specifically, Burns, Nettelbeck and White (1998) examined the psychophysical functions associated with various aspects of the IT task. They demonstrated that IT cannot be rendered completely immune to the contamination of performance by the use of subtle visual cues, including the detection of apparent movement. The nature of the IT task is such, therefore, that the detection of these visual cues is demanded under severe time constraints: it is plausible, then, that IT provides a measure of speed of visualisation (VSp); this construct itself is subsumed by general speed of processing (Gs).

We turn now to the question of whether IT and reaction time measures share common variance with IQ. First, it is clear that the variance both IT and CRT DTs share with IQ is not common although both measures fit into a hierarchical model of intelligence under a broad speed of processing factor. The situation with respect to OMO DT is more complex. As noted above, studies on IT and OMO DT have been equivocal; moreover, whether OMO DT measures fluid ability or not is unclear. Burns and Nettelbeck (in press) reported IT and OMO DT as independent with the latter being a measure of Gf. Roberts and Stankov (1999) also suggested that DT from a task they considered as equivalent to OMO DT was correlated with fluid ability. The analyses here, however, show IT and OMO DT loading on the VSp factor. The communality of OMO DT was quite low; it is therefore suggested that only a small part of variance in this measure is related to speed of processing abilities. Specifically, OMO DT provides measures of visualisation speed and DT but, nonetheless, mainly measures fluid intelligence. Such a suggestion fits with the extant literature on OMO DT. 
The data presented here are highly supportive of the model of mental speed proposed by Roberts and Stankov (1999). Their analysis revealed five group factors: MT, DT, Gs (speed of doing perceptual tasks), Tv/a (speed of doing tasks involving Gv and Ga) and Tir (speed of doing inductive reasoning tasks). The first four of these factors were confirmed in the current study. Because Roberts and Stankov included inductive reasoning tasks in their battery and measured the speed of performance on these tasks, an inductive reasoning speed factor (Tir) emerged in their analysis. Correspondingly, we could have labeled our CDS factor Tf/wm (speed of doing tasks involving Gf and working memory) but the swaps variables alone were too narrow to define such a factor. The inference drawn is that if a diverse battery was administered, there may be speed factors associated with each of the second order factors defined in Gf - Gc theory. Other models of mental speed (e.g., Horn, 1988; Carroll, 1993) have aspects that are inconsistent with the Roberts and Stankov model. Horn (1988) described two-second order speed factors: CDS (speed at correctly solving more difficult cognitive tasks) and Gs (number correct scores from simple tasks such as perceptual tasks). He showed that these two factors were correlated at a low level. Carroll (1993) also proposed two second-order speed factors: broad cognitive speediness and processing speed. The cognitive speediness factor is defined by speed of test-taking, perceptual speed and numerical facility; the processing speed factor is defined by simple and choice decision time, mental comparison speed and semantic processing speed. Thus, the processing speed factor is thought of as a DT factor. It can be seen, therefore, that Carroll's analysis differentiated speed at performing the psychometric tests from the more basic processing speed tests. We conclude that the differences across these models are largely ones of emphasis. We also alluded to the uncertainty of the order of analysis at which these various factors emerge and fit into the hierarchy of cognitive abilities. The narrow focus of the current study does not allow resolution of these issues (see also Keith, 1997, Roberts \& Stankov, 1999).

The relationship between IT and intelligence has been clarified to some degree by the current study. IT is related to intelligence because it is a measure of speed of processing. Speed of 
processing abilities are factorially complex, and IT provides a very specific measure of visualisation speed (although it may not be a strong marker of this construct). Future studies in our laboratory will focus on test batteries including multiple tests of visualisation abilities. This will allow us to define more adequately the visualisation speed factor and confirm that, at this detailed level of analysis, IT is independent from a perceptual speed factor.

Finally, it is worth noting the low communality of IT (.206) in this battery. The corresponding communality, in a much broader battery of tests, reported by Burns and Nettelbeck (in press) was .337, that is, somewhat higher but still indicative of marked specificity. Moreover, in that study two versions of IT, one presented on a video monitor and the other using LEDs, correlated only $r=.29$. Inspection time does not measure fluid abilities. It must be inferred that IT has high specificity (given that its reliability is moderately high); therefore, manipulations of IT, and even longitudinal observations on IT, cannot assume that any changes (or stationarity) in the measure involve those aspects of IT variance that relate to intelligence and such issues remain to be tested empirically. These observations warrant careful consideration by those who would use IT as a marker for Spearman's $g$ in various studies on the biological basis of intelligence. 


\section{References}

Arbuckle, J.L. (1999). AMOS 4.0 users' guide. Chicago, IL; SPSS Inc.

Bates, T. C., \& Eysenck, H. J. (1993). Intelligence, inspection time and decision time. Intelligence, $17,523-531$.

Brand, C. R., \& Deary, I. J. (1982). Intelligence and “inspection time”. In H. J. Eysenck (Ed.), A model for intelligence (pp. 133 - 148). New York: Springer-Verlag

Burns, N. R., \& Nettelbeck, T. (in press). Inspection time in the structure of cognitive abilities: Where does IT fit? Intelligence.

Burns, N. R., Nettelbeck, T., \& Cooper, J. (1999). Inspection time correlates with general speed of processing but not with fluid ability. Intelligence, 27, 37-44.

Burns, N. R., Nettelbeck, T., \& White, M. (1998). Testing the interpretation of inspection time as a measure of speed of sensory processing. Personality and Individual Differences, 24, 25-39.

Carroll, J. B. (1991). No demonstration that $g$ in not unitary, but there is more to the story: Comment on Kranzler and Jensen. Intelligence, 15, 423-436.

Carroll, J. B. (1993). Human cognitive abilities: A survey of factor analytic studies. NY: Cambridge University Press.

Carroll, J. B. (1995). On methodology in the study of cognitive abilities. Multivariate Behavioural Research, 30, 429-452.

Crawford, J. D. (1988). Intelligence, task complexity and tests of sustained attention. Unpublished $\mathrm{PhD}$ Thesis, The University of New South Wales.

Crawford, J. R., Deary, I. J., Allan, K. M., \& Gustafsson, J. (1998). Evaluating competing models of the relationship between inspection time and psychometric intelligence. Intelligence, 26, $27-42$.

Deary, I.J. (1993). Inspection time and WAIS-R IQ subtypes: A confirmatory factor analysis study. Intelligence, 17, 223-236. 
Deary, I. J. (2000). Looking down on human intelligence: From psychometrics to the brain. Oxford: OUP.

Evans, G., \& Nettelbeck, T. (1993). Inspection time: a flash mask to reduce apparent movement effects. Personality and Individual Differences, 15, 91-94.

Frearson, W., Barrett, P., \& Eysenck, H. J. (1988). Intelligence, reaction time and the effects of smoking. Personality and Individual Differences, 9, 497-517.

Frearson, W. \& Eysenck, H.J. (1986). Intelligence, reaction time (RT) and a new 'odd-man-out' RT paradigm. Personality and Individual Differences, 7, 807-817.

Grudnik, J.L. \& Kranzler, J.H. (2001). Meta-analysis of the relationship between intelligence and inspection time. Intelligence, 29, 523-535.

Horn, J. (1988). Thinking about human abilities. In J. R. Nesselroade, \& R. B. Cattell (Eds.), Handbook of Multivariate Experimental Psychology (2nd ed., pp. 645-685). NY: Plenum. Horn, J. (1989). Remodelling old models of intelligence. In B. B Wolman (Ed.), Handbook of Intelligence: Theories, Measurements, and Applications (pp. 267-300). NY: Wiley.

Horn, J. L. \& Cattell, R. B. (1966). Refinement and test of the theory of fluid and crystallized intelligence. Journal of Educational Psychology, 57, 253-270.

Horn, J., \& Noll, J. (1994). A system for understanding cognitive capabilities: A theory and the evidence on which it is based. In D. K. Detterman (Ed.), Current topics in human intelligence Vol. 4: Theories of intelligence (pp. 151-203). Norwood, NJ: Ablex.

Jensen, A. R. (1982). Reaction time and psychometric g. In H. J Eysenck (Ed.), A Model for Intelligence (pp. 93-132). NY: Springer-Verlag.

Jensen, A. R., \& Munro, E. (1979). Reaction time, movement time and intelligence. Intelligence, 3, $121-126$.

Kaiser, H. F. (1981). A revised measure of sampling adequacy for factor-analytic data matrices. Educational and Psychological Measurement, 41, 379-381. 
Keith, T. Z. (1997). Using confirmatory factor analysis to aid in understanding the constructs measured by intelligence tests. In D.P. Flanagan, J.L. Genshaft and P.L. Harrison (Eds.), Contemporary intellectual assessment: Theories, tests, and issues (pp.373-402). NY: Guilford Press.

Kline, P. (1991). Intelligence: The psychometric view. London: Routledge.

Kranzler, J. H., \& Jensen, A. R. (1989). Inspection time and intelligence: A meta-analysis. Intelligence, 13, 329-347.

Kranzler, J. H., \& Jensen, A. R. (1991). The nature of psychometric g: Unitary process or a number of independent processes? Intelligence, 15, 397-422.

Mackintosh, N. J. \& Bennett, E. S. (2002). IT, IQ and perceptual speed. Personality and Individual Differences, 32, 685-693.

Nettelbeck, T. (1987). Inspection time and intelligence. In P. A Vernon (Ed.), Speed of Information Processing and Intelligence (pp. 295-346). NJ: Ablex.

Roberts, R.D., \& Stankov, L. (1999). Individual differences in speed of mental processing and human cognitive abilities: Toward a taxonomic model. Learning and Individual Differences, $11,1-120$.

Sattler, J. M. (1982). Age effects on Wechsler Adult Intelligence Scale - Revised Tests. Journal of Consulting and Clinical Psychology, 50, 785-786

Stankov, L., \& Roberts, R. D. (1997). Mental speed is not the 'basic' process of intelligence. Personality and Individual Differences, 22, 69-84.

Vickers, D., \& Smith, P. L. (1986). The rationale for the inspection time index. Personality and Individual Differences, 7, $609-623$.

Wechsler, D. (1981). WAIS-R Manual: Wechsler Adult Intelligence Scale-Revised. The Psychological Corporation: New York.

Wetherill, G. B., \& Levitt, H. (1965). Sequential estimation of points on a psychometric function. British Journal of Mathematical and Statistical Psychology, 18, 1-10. 
Wittenborn, J. R. (1943). Factorial Equations for Tests of Attention. Psychometrika, 8, 19-35.

This is a post-print of an article published under the same title by Elsevier Ltd in the journal Personality and Individual Difference (vol. 35, no. 3, 2003, pp. 713-724). The published version can be found at: 


\section{Acknowledgement}

We thank Professors Ted Nettelbeck and Lazar Stankov for their comments on an earlier version of this paper. Preparation of the manuscript was partially supported by Australian Research Council Discovery Grant DP0211113. 
Table 1

Correlation matrix, means and standard deviations for 18 variables $(N=102)$

\begin{tabular}{|c|c|c|c|c|c|c|c|c|c|c|c|c|c|c|c|c|c|c|c|}
\hline$\underline{1}$ & DS & $\underline{1}$ & $\underline{2}$ & $\underline{3}$ & $\underline{4}$ & $\underline{5}$ & $\underline{6}$ & $\underline{7}$ & $\underline{8}$ & $\underline{9}$ & $\underline{10}$ & $\underline{11}$ & $\underline{12}$ & $\underline{13}$ & $\underline{14}$ & $\underline{15}$ & $\underline{16}$ & $\underline{17}$ & $\underline{18}$ \\
\hline$\underline{2}$ & MR & .421 & & & & & & & & & & & & & & & & & \\
\hline$\underline{3}$ & $\mathrm{CO}$ & .631 & .368 & & & & & & & & & & & & & & & & \\
\hline$\underline{4}$ & IT & -.208 & -.203 & -.148 & & & & & & & & & & & & & & & \\
\hline$\underline{5}$ & $2 \mathrm{DT}$ & -.147 & -.304 & -.106 & .133 & & & & & & & & & & & & & & \\
\hline$\underline{6}$ & $2 \mathrm{MT}$ & .002 & -.084 & -.056 & .033 & .395 & & & & & & & & & & & & & \\
\hline$\underline{7}$ & $4 \mathrm{DT}$ & -.193 & -.274 & -.069 & .110 & .830 & .317 & & & & & & & & & & & & \\
\hline$\underline{8}$ & $4 \mathrm{MT}$ & .001 & -.083 & -.109 & .075 & .247 & .837 & .230 & & & & & & & & & & & \\
\hline$\underline{9}$ & $8 \mathrm{DT}$ & -.129 & -.203 & -.050 & .119 & .716 & .328 & .784 & .209 & & & & & & & & & & \\
\hline$\underline{10}$ & $8 \mathrm{MT}$ & .012 & -.024 & -.066 & .079 & .175 & .767 & .209 & .879 & .145 & & & & & & & & & \\
\hline$\underline{11}$ & OMO DT & -.167 & -.340 & -.141 & .354 & .435 & .278 & .499 & .232 & .565 & .152 & & & & & & & & \\
\hline$\underline{12}$ & OMO MT & -.115 & -.106 & -.188 & .131 & .264 & .663 & .212 & .751 & .164 & .729 & .147 & & & & & & & \\
\hline$\underline{13}$ & Triplet 1 & -.407 & -.352 & -.413 & .293 & .224 & .069 & .166 & .041 & .213 & -.007 & .304 & .095 & & & & & & \\
\hline$\underline{14}$ & Triplet 2 & -.435 & -.432 & -.424 & .321 & .238 & .077 & .240 & .090 & .194 & .074 & .465 & .184 & .652 & & & & & \\
\hline$\underline{15}$ & Triplet 3 & -.338 & -.395 & -.287 & .161 & .341 & .043 & .306 & -.005 & .276 & -.059 & .354 & .068 & .459 & .531 & & & & \\
\hline$\underline{16}$ & 2-Swap & -.566 & -.370 & -.544 & .274 & .240 & .010 & .248 & .048 & .175 & .046 & .268 & .115 & .406 & .425 & .459 & & & \\
\hline$\underline{17}$ & 3-Swap & -.536 & -.351 & -.464 & .252 & .180 & -.019 & .197 & .029 & .117 & .033 & .260 & .099 & .302 & .355 & .361 & .833 & & \\
\hline \multirow[t]{3}{*}{$\underline{18}$} & 4-Swap & -.545 & -.398 & -.444 & .216 & .267 & .071 & .262 & .099 & .183 & .076 & .311 & .152 & .280 & .357 & .379 & .887 & .904 & \\
\hline & Mean & 65.3 & 52.1 & 32.8 & 62.1 & 282.3 & 147.1 & 292.4 & 150.2 & 317.3 & 155.9 & 569.3 & 169.6 & 850.5 & 1157.3 & 3014.7 & 11771 & 17746 & 23634 \\
\hline & SD & 10.7 & 12.1 & 6.5 & 16.2 & 32.4 & 24.5 & 31.8 & 24.2 & 37.7 & 25.4 & 128.8 & 34.9 & 195.7 & 269.6 & 1071.2 & 3091.8 & 4435.4 & 6540.5 \\
\hline
\end{tabular}

Note: Significant correlations $(\mathrm{p}<0.05)$ are shown in bold.

Digit symbol, mental rotation and cross out are number correct scores, all other measures are ms 
Table 2

Factor structure matrix for oblique rotation of the five-factor solution, correlation matrix for the five factors and their loadings on the second order general speed factor (Gs)

\begin{tabular}{|c|c|c|c|c|c|}
\hline & MT & DT & VSp & PSp & CDS \\
\hline Digit Symbol & .010 & .040 & -.005 & .548 & .061 \\
\hline Mental Rotation & -.007 & .156 & .209 & .319 & -.016 \\
\hline Cross Out & .136 & -.060 & .000 & .605 & -.014 \\
\hline IT & .025 & -.021 & .350 & .059 & .037 \\
\hline $2 \mathrm{DT}$ & .040 & .788 & -.012 & .052 & -.004 \\
\hline $2 \mathrm{MT}$ & .770 & .202 & -.001 & .013 & -.047 \\
\hline $4 \mathrm{DT}$ & -.008 & .863 & -.042 & -.002 & .038 \\
\hline $4 \mathrm{MT}$ & .919 & .022 & .016 & .010 & .008 \\
\hline $8 \mathrm{DT}$ & -.028 & .773 & .076 & -.036 & -.021 \\
\hline $8 \mathrm{MT}$ & .889 & -.028 & -.015 & -.026 & .045 \\
\hline OMO DT & .018 & .357 & .469 & -.032 & .014 \\
\hline OMO MT & .757 & .019 & .006 & .138 & -.019 \\
\hline Triplet 1 & -.024 & .026 & .471 & .472 & -.233 \\
\hline Triplet 2 & .017 & -.003 & .636 & .466 & -.229 \\
\hline Triplet 3 & -.113 & .194 & .326 & .275 & -.027 \\
\hline 2-Swaps & -.013 & .012 & .044 & .143 & .554 \\
\hline 3-Swaps & -.021 & -.041 & .004 & .010 & .677 \\
\hline 4-Swaps & .026 & .043 & -.026 & .002 & .679 \\
\hline & $\underline{\mathrm{MT}}$ & $\underline{\mathrm{DT}}$ & VSp & $\underline{\mathrm{PSp}}$ & $\underline{\mathrm{GS}}$ \\
\hline$\underline{\mathrm{MT}}$ & & & & & .090 \\
\hline$\underline{\mathrm{DT}}$ & .239 & & & & .315 \\
\hline$\underline{\mathrm{VSp}}$ & .146 & .315 & & & .462 \\
\hline$\underline{\mathrm{PSp}}$ & -.156 & .142 & .221 & & .618 \\
\hline$\underline{\mathrm{CDS}}$ & .037 & .228 & .412 & .644 & .944 \\
\hline
\end{tabular}

Note: Highest loadings for each variable are shown in bold.

MT is movement time; DT is decision time; VSp is visualisation speed; PSp is perceptual speed; CDS is correct decision speed; Gs is general speed of processing

This is a post-print of an article published under the same title by Elsevier Ltd in the journal Personality and Individual Difference (vol. 35, no. 3, 2003, pp. 713-724). The published version can be found at: 